\title{
Multi Resolution Analysis for Consonant Classification in Noisy Environments
}

\author{
T M Thasleema ${ }^{1}, \mathrm{~N}$ K Narayanan ${ }^{2}$ \\ Department of Information Technology, Kannur University, Kerala, India, 670567 \\ e-mail:thasnitm1@ hotmail.com¹, nknarayanan@gmail.com ${ }^{2}$
}

\begin{abstract}
This paper investigates on the use of Wavelet Transform (WT) to model and recognize the utterances of Consonant - Vowel (CV) speech units in noisy environments. The peculiarity of the proposed method lies in the fact that using WT, non stationary nature of the speech signal can be accurately considered. A hybrid feature extraction namely Normalized Wavelet Hybrid Feature (NWHF) using the combination of Classical Wavelet Decomposition (CWD) and Wavelet Packet Decomposition (WPD) along with z-score normalization technique are studied here. $\mathrm{CV}$ speech unit recognition tasks performed for both noisy and clean speech units using Artificial Neural Network (ANN) and k Nearest Neighborhood $(\mathrm{k}-\mathrm{NN})$ are also presented. The result indicates the robustness of the proposed technique based on WT in additive noisy condition.
\end{abstract}

Index Terms - Wavelet Transform, Normalized Wavelet Hybrid Features, Daubechies Wavelet, k - Nearest Neighborhood, Artificial Neural Network

\section{INTRODUCTION}

Speech recognition research has a history of more than 50 years. With the advancement of powerful computers and robust algorithms, Automatic Speech Recognition (ASR) has undergone a great amount of progress over the last few years. For reasons ranging from technological curiosity about the mechanisms for mechanical realization of human speech capabilities, to the desire to automate simple tasks naturally requiring human-machine interactions, research in ASR and speech synthesis by machine has attracted a great deal of attention over the past six decades. The earliest attempt to build an ASR system where made in 1950s' based on acoustics phonetics features. These systems relied on spectral measurements using spectrum analysis and pattern matching to make recognition decisions on tasks such as vowel recognition [1]. Filter bank analysis was also implemented in some systems to provide spectral information. Several basic speech recognition ideas were emerged in the 1960 s'. Zero - Crossing Analysis (ZCA), speech segmentation, dynamic time aligning and tracking etc algorithms were proposed during this period [2]. In the 1970's, speech recognition research achieved major milestones. Isolated word recognition systems become possible using Dynamic Time warping (DTW). Linear Predictive Coding (LPC) was extended from speech coding into speech recognition systems based on
LPC spectral parameters. IBM came out with the effort of large vocabulary speech recognition system in the 70s', which turned out to be highly successful and had a great impact on speech recognition research. AT \& T Bell Labs also began to making truly speaker independent speech recognition systems by studying clustering algorithms for creating speaker independent patterns. In the 1980's connected word recognition system were devised based on algorithms that concatenated isolated words for recognition. Hidden Markov Models (HMM) are widely used in almost all researchers after mid-1980s. In the late 1980s, Neural Networks were also introduced to problems in speech recognition as a signal classification technique.

There have been a lots of popular attempts carried out towards ASR which kept the research in this area vibrant. Generally a speech recognition system tries to identify the basic unit in any language, phonemes or words which can be compiled into text [3]. The potential applications of ASR include computer speech to text dictation, automatic call routing and machine language translation. ASR is a multi disciplinary area that draws theoretical knowledge from mathematics, physics and engineering. Specific topics include signal processing, information theory, random processes, machine learning, pattern recognition, psychoacoustics and linguistics.

The present research work is motivated by the knowledge that only little attempts were rendered for the automatic speech recognition of $\mathrm{CV}$ speech unit in Indian languages like Hindi, Tamil, Bengali, Marathi Chinese etc and very less works have been found to be reported in the literature on the recognition of $\mathrm{CV}$ speech units in Malayalam, which is the principal language of South Indian state of Kerala. Very few research attempts were reported so far in the area of Malayalam vowel recognition. So more basic research works are essential in the area of Malayalam CV speech unit recognition.

Malayalam is one of the major languages from Dravidian language family and other major languages include Kannada, Tamil, Telugu, Tulu and Konkani. Malayalam is the principal language of the South Indian state of Kerala and also of the Lakshadweep Islands off the west coast of India spoken by about 36 million people [4]. Malayalam language now contains $51 \mathrm{~V} / \mathrm{CV}$ units that include 15 short and long vowel sounds and the remaining 36 basic consonant sounds. The earlier writing style of the Malayalam is now substituted with a 
new style from 1981. Compared to Malayalam and all other Indian languages Tamil seems to be different in the sense that Tamil doesn't have aspirated sounds and thus the pronunciation is different from other Dravidian language structures. Tamil contains only 'kharam' and 'anunasikam' sounds and thus the script used to represent 'mridu' sounds are using 'kharam'. In Tamil the pronunciation of 'kharam' lies in the range between 'kharam' and 'mridu' compared to Malayalam. For example the word 'ganapathi' pronounced and scripted as 'kanapathi'. In Bengali the pronunciation of the vowel ' $a$ ' is replaced with 'au'. Due to lineage of Malayalam to both Sanskrit and Tamil, Malayalam language structure has the largest number of phonemic utterances among the Indian languages. Malayalam script includes letters capable of representing all the phoneme of Sanskrit and all Dravidian languages. A unique property of Malayalam is 'chillukal' which is derived from the basic consonant units.

A consonant can be defined as a unit sound in spoken language which is described by a constriction or closure at one or more points along the vocal tract. According to Peter Ladefoged, consonants are just ways of beginning or ending of vowels [5]. Consonants are made by restricting or blocking the airflow in some way and each consonant can be distinguished by where this restriction is made [6]. The point of maximum restriction is called the place of articulation of a consonant. A consonant also can be distinguished by how the restriction is made. For example, where there is a complete stoppage of air or only a partial blockage of it. This feature is called the manner of articulation of a consonant. The combination of place and manner of articulation is sufficient to uniquely identify a consonant. In the present work, all the experiments are carried out using 36 Malayalam CV speech unit database uttered by 96 different speakers. For the recognition experiments, database is divided into five different phonetic classes based on the manner of articulation of the consonants as given in table 1 .

Table 1: Malayalam CV unit classes

\begin{tabular}{|c|c|}
\hline Class & Sounds \\
\hline Unaspirated & $\begin{array}{l}/ \mathrm{ka} /, / \mathrm{ga} /, / \mathrm{cha} /, / \mathrm{ja} /, / \mathrm{ta} /, / \mathrm{da} /, / \mathrm{tha} /, \\
/ \mathrm{d}_{\mathrm{h}} \mathrm{a} /, / \mathrm{pa} /, / \mathrm{ba} /\end{array}$ \\
\hline Aspirated & $\begin{array}{l}\text { /kha/,/gha/,/chcha/,/jha/, /tta/, /dda/, } \\
\text { /ththa/, /dha/,/pha/, /bha/ }\end{array}$ \\
\hline Nasals & /nga/,/na/,/nna/,/na/,/ma/ \\
\hline Approximants & /ya/,/zha/,/va/,/lha/,/la/ \\
\hline Fricatives & /sha/,/shsha/,/sa/,/ha/,/ra/,/rha/ \\
\hline
\end{tabular}

Since human speech is highly dynamic in nature, in order to achieve a reliable representation of the speech signal in the time - frequency plane a multi resolution approach is needed. Wavelet Transform (WT) is a tool for Multi Resolution Analysis (MRA) which can be used to efficiently represent the speech signal in the time - frequency plane. There have been lots of works reported in the literature using WT for the feature extraction process [7][8][9]. The objective of the present work is to model Malayalam CV speech unit waveforms using WT based Normalized Wavelet Hybrid Feature (NWHF) extraction technique in speaker independent environments. Classifications are carried out using Artificial Neural Networks (ANN) and k - Nearest Neighborhood (k-NN) algorithms. The performance of the present method is discussed in noisy environments. The rest of the paper is organized as follows. Section II of this paper gives an overview on wavelets and wavelet transform and in section II.1 a brief description on subband coding and multi resolution analysis. Section III.1 and III.2 describes the wavelet decomposition approaches namely Classical Wavelet Decomposition (CWD) and Wavelet Packet Decomposition (WPD) respectively. In section IV, NWHF based feature extraction technique for the Malayalam CV speech unit is explained. Section V.1 and V.2 describes classification using $\mathrm{k}-\mathrm{NN}$ and ANN classifiers. Section VI presents the simulation experiments conducted using Malayalam CV speech unit database and reports the recognition results obtained using ANN and k - NN classifiers. Finally section VII gives the conclusion and direction for future work.

\section{WAVELETS AND WAVELET TRANSFORM}

Certain ideas of wavelet theory appeared quite a long time ago [10]. Over the last decades wavelet analysis has turned to be a standard technique in the areas of geophysics, meteorology, audio signal processing and image compression [11][12][13]. Wavelet transform can be defined as the transformation of the signal under analysis into another representation which presents the signal in a more useful form [14]. Mathematically a wavelet can be denoted as

$\psi_{a, b}(t)=\frac{1}{\sqrt{|a|}} \psi\left(\frac{t-b}{a}\right)$

Where $b$ is the location parameter (translation) and $a$ is the scaling parameter (dilation). Wavelet functions are excellent mathematical tool in the time - frequency analysis of both one dimensional and two dimensional signals. Various important classes of wavelets include smooth wavelets, compactly supported wavelets, symmetric and non - symmetric wavelets, orthogonal and bi orthogonal wavelets etc. Based on the definition of wavelet, wavelet transform of a signal $f(t)$ can be mathematically represented as

$$
\begin{aligned}
& W_{(a, b)}=\int_{-\infty}^{\infty} f(t) \psi_{a, b} d t \\
& \text { ie. } W_{(a, b)}=\int_{t=-\infty}^{\infty} f(t) \frac{1}{\sqrt{|a|}} \psi\left(\frac{t-b}{a}\right)
\end{aligned}
$$

Wavelet transforms are proved to be a very important and useful tool for signal and image processing [14]. Several applications of wavelet transform are already proposed in the literature [15] [16]. Coifman and Maggioni had proposed wavelets based on diffusion 
operators in their work [17]. Hammond et al introduced wavelets on graph via spectral graph theory [18]. Kadambe has made a study on pitch detection algorithm for speech signal using wavelet transform [19]. O Farook et al had proposed in their research work the use of wavelet based feature extraction technique for Hindi phoneme recognition and proved that the proposed technique achieves a better performance over MFCC based features [20].

The Discrete Wavelet Transform (DWT) has been treated as a Natural Wavelet Transform (NWT) for discrete time signals by different authors [21][22]. For computing the wavelet coefficients several discrete algorithms have been established [23]. Daubechies and some others had invented DWT peculiarly designed for analyzing finite set of observations over the set of scales using dyadic discretization [24][25]. As Daubechies mentioned in his work, DWT can be interpreted as continuous wavelets with discrete scale and translation factors. The WT is then evaluated at discrete scale and translation. The discrete scaling is expressed as $a=a_{0}{ }^{j}$ where $\mathrm{i}$ is an integer and $\mathrm{a}_{0}>1$. The discrete translation factor is expresses as $b=k b_{0} a_{0}{ }^{j}$ where $k$ is an integer. Thus DWT can be mathematically represented as

$$
D W T_{j, k}=C W T\left\{f(t) ; a=a_{0}^{j}, b=k b_{0} a_{0}^{j} j, k \in z\right\}
$$

where $\mathrm{z}$ is the set of integers.

Most often DWT coefficients are usually sampled from CWT on a dyadic grid, i.e., $\mathrm{a}_{0}=2$ and $\mathrm{b}_{0}=1$.

Then wavelet in eqn (1) can be written as

$$
\psi_{j, k}(t)=2^{-j / 2} \psi\left(2^{-j}(t-k)\right)
$$

and eqn(3) becomes

$$
W_{j, k}(t)=\sum_{j} \sum_{k} f(k) 2^{-j / 2} \psi\left(2^{-j} t-k\right)
$$

More effective implementation of DWT is obtained using MRA based subband coding technique discussed below.

\section{A. Multi Resolution Analysis and Subband Coding}

Multi Resolution Analysis (MRA) is an effective tool for interpreting the information content of a signal. A MR representation produces a simple hierarchical model to develop the algorithms for the time-frequency analysis of the signal. At different resolution, the details of the speech signal generally characterize vocal characteristics of the speech signal. Each signal to be characterized is analyzed at a coarse resolution and then gradually increases the resolution. Thus MRA can be defined as a technique that permits us to analyze the signal in multiple frequency bands. Two important existing approaches for MRA are the Laplacian Pyramid and Subband coding. To compute the signal details at different resolution Burt and Crowley have introduced the pyramidal implementation [26][27]. In this method the details at different resolutions are regrouped into a pyramidal structure called Laplacian Pyramid. In Laplacian Pyramidal data structures, data at different levels are correlated and this might be a difficulty. Distinctive form Laplacian Pyramid, Subband Coding is an efficient Multi Resolution Spectral Analysis tool for speech signal processing. The main objective of subband coding is to segment the speech signal spectrum into independent subbands in order to treat the subbands individually for different purposes [28].

\section{WAVELET DECOMPOSITIONS}

The main advantage of DWT over FT is the multi resolution analysis of signals with localization on both time and frequency. In the present work we utilize these characteristics of wavelet transform using two major wavelet decomposition techniques for $\mathrm{CV}$ speech unit recognition., viz CWD and WPD.

\section{A. Classical Wavelet Decomposition (CWD)}

In Classical Wavelet Decomposition (CWD), the decomposition of the signal is obtained by successive high pass and low pass filtering of the time domain signal. The original signal is first passed through a halfband highpass filter and a lowpass filter. This is the first level decomposition. This decomposition halves the time resolution since only half the number of samples now characterizes the entire signal. At every level, the filtering and subsampling will result in half the number of samples (and hence half the time resolutions) and half the frequency band spanned (and hence doubles the frequency resolutions). In first level decomposition the speech signal is split into an approximation and detail signal according to high pass and low pass filtering. The approximation is then again split into a second level approximation and detail signal and the process is continued for $\mathrm{n}^{\text {th }}$ level. CWD based approximation signal Plot of fifth level decomposition for the speech sound $/ \mathrm{ka} /$ is plotted in figure 1 .
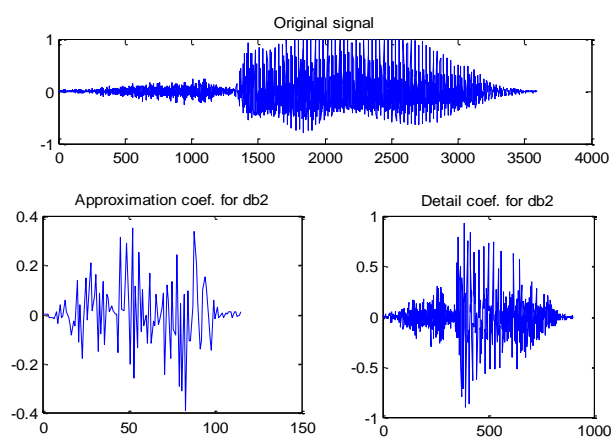

Figure 1: CWD plot for the sound $/ \mathrm{ka} /$ using $\mathrm{db} 2$

\section{B. Wavelet Packet Decomposition (WPD)}

Wavelet Packet Decomposition (WPD) is also known as Wavelet Packets (WP) is a wavelet transform in which the signal is passed through more number of filters than CWD. In CWD, each level is calculated by passing the previous approximation signal through a high pass and low pass filters while is WPD, both 
approximation and detailed signals are decomposed. WPD Plot for the fifth level of decomposition for the speech sound $/ \mathrm{ka} /$ is given in figure 2 .

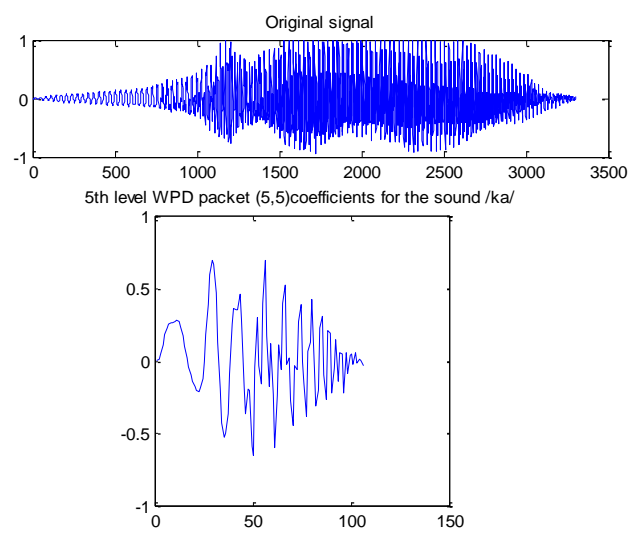

Figure 2: WPD plot for sound $/ \mathrm{ka} / \mathrm{using} \mathrm{db} 2$

\section{NORMALIZED WAVELET HYBRID FEATURE EXTRACTION}

Normalized Wavelet Hybrid Feature (NWHF) vector for the present work is generated using CWD and WPD method. The process for extracting NWHF feature vector is described below.

In the first step, the sound signal is made to undergo recursively to decompose into $\mathrm{k}^{\text {th }}$ level of resolutions; depending upon the approximation coefficient matrix at this $\mathrm{k}^{\text {th }}$ level is sufficient to classify each speech sound to the corresponding sound class and carries enough information content to describe sound characteristics.

Let $A_{k}$ represents this approximation matrix at decomposition level $\mathrm{k}$, which can be written as

$$
A_{k}=\left[\begin{array}{cccc}
A_{1,1} & A_{1,2} & \ldots . & A_{1, n} \\
A_{2,1} & A_{2,2} & \ldots . & A_{2, n} \\
\ldots & \ldots & \ldots . & \ldots . \\
A_{k, 1} & A_{k, 2} & \ldots . & A_{k, n}
\end{array}\right]
$$
is,

Then, the first component of NWHF feature vector $v_{1}$

$$
v_{1}=\bigcup_{i=1}^{k} \bigcup_{j=1}^{n}\left\{A_{i, j}\right\}
$$

In the second step, for Wavelet Packet Decomposition, decompose each speech segment using $\mathrm{k}^{\text {th }}$ level of resolutions for the best level of wavelet packet decomposition tree. The first coefficient matrix at the best level tree contains enough information to represent the given input consonant $\mathrm{CV}$ speech unit without loss of much speech features. Let $m$ represent mean of one row vector in the coefficient matrix then the WPD feature vector $\boldsymbol{v}_{\mathbf{2}}$ is given by

$$
v_{2}=m_{i}, \mathrm{i}=1,2, \ldots . . \mathrm{m}
$$

(where ' $\mathrm{m}$ ' is the number of rows in the best level coefficient matrix).
In the third step we combined $v_{1}$ and $v_{2}$ to fusion CWD and WPD coefficients.

$V=\bigcup_{i=1}^{2}\left\{v_{i}\right\}$

Then the final feature vector $\mathrm{F}$ after $\mathrm{Z}$-score normalization is given as

$\boldsymbol{F}_{=} \frac{V-\mu(V)}{\sigma(V)}$

Where $\mu$ and $\sigma$ represents mean and variances with respect to the feature vector $\mathrm{V}$

The feature vector of size 20 is estimated by concatenating CWD and WPD features for hybrid feature vectors. The NWHF vector for different speaker shows the identity of the same sound so that an efficient feature vector can be formed using NWHF vectors. The feature vector obtained for different speech unit seems to be distinguishable, giving discriminate feature vector for speech unit recognition.

\section{CLASSIFICATION}

Pattern recognition can be defined as the categorization of input data into identifiable classes via significant feature extraction of the data from the background of irrelevant details. This is the field concerned with machine recognition of meaningful regularities in noisy or complex environments [29]. Nowadays pattern recognition is an integral part of most intelligent systems built for decision making. In the present study two widely used approaches for pattern recognition problems namely statistical pattern classifier $(\mathrm{k}-\mathrm{NN})$ and connectionist approaches (ANN) are used The task of a classifier component proper of a full system is to use the feature vector provided by the feature extractor to assign the object to a category [30].

\section{A. $\quad k$-Nearest Neighborhood}

Pattern classification using distance function is an earliest concept in pattern recognition [31] [32]. Here the proximity of an unknown pattern to a class serves as a measure of its classifications. $\mathrm{k}-\mathrm{NN}$ is a well known non - parametric classifier, where a posteriori probability is estimated from the frequency of the nearest neighbors of the unknown pattern [33]. For classifying each incoming pattern $\mathrm{k}-\mathrm{NN}$ requires an appropriate value of $\mathrm{k}$. A newly introduced pattern is then classified to the group where the majority of $\mathrm{k}$ nearest neighbor belongs [34]. Hand proposed an effective trial and error approach for identifying the value of $\mathrm{k}$ that incurs highest recognition accuracy [35]. Various pattern recognition studies with highest performance accuracy are also reported based on these classification techniques [36] [37] [38].

Consider the cases of $\mathrm{m}$ classes $\mathrm{c}_{\mathrm{i}}, \mathrm{i}=1,2, \ldots \ldots \mathrm{m}$, and a set of $\mathrm{N}$ samples pattern $\mathrm{y}_{\mathrm{i}}, \mathrm{i}=1,2, \ldots \ldots \mathrm{N}$ whose classification is priory known. Let $\boldsymbol{x}$ denote an arbitrary 
incoming pattern. The nearest neighbor classification approach classifies $\boldsymbol{x}$ in the pattern class of its nearest neighbor in the set $y_{\mathrm{i}}$

$$
\begin{aligned}
& \text { i.e. If }\left\|x-y_{j}\right\|^{2}=\min \left\|x-y_{i}\right\|^{2} \text {, where } 1 \leq \mathrm{i} \leq \mathrm{N} \\
& \text { then } \boldsymbol{x} \text { in } \mathrm{c}_{\mathrm{j}}
\end{aligned}
$$

This is $1-\mathrm{NN}$ rule since it employs only one nearest neighbor to $\boldsymbol{x}$ for classification. This can be extended by considering $\mathrm{k}$ - Nearest Neighbors to $\boldsymbol{x}$ and using a majority - rule type classifier.

\section{B. Artificial Neural Network}

In recent years, neural networks have been successfully applied in many of the pattern recognition and machine learning systems [39] [40] [41]. ANN is an arbitrary connection of simple computational elements [42]. In other words, ANN's are massively parallel interconnection of simple neurons which are intended to abstract and model some functionalities of human nervous system [43][44]. Neural networks are designed to mimic the human brain in order to emulate the human performance and there by function intelligently [45]. Neural network models are specified by the network topologies, node or computational element characteristics, and training or learning rules. The three well known standard topologies are single or multilayer perceptrons, Hopfield or recurrent networks and Kohonen or self organizing networks.

Various Neural Network (NN) learning algorithms have been evolved in the past years. In all these algorithms, a set of rules defines the evolution process undertaken by the synaptic connections of the networks, thus allowing them to learn how to perform the specified tasks. In the present study Back Propagation Learning Algorithm (BPLA) is used for classifying Consonant Vowel (CV) units.

The BPLA is the most popular method for NN training and it has been used to solve numerous real life problems. In a Feed Forward Multi Layer Perceptron (FFMLP), BP algorithm performs iterative minimization of a cost function by making weight connection adjustments according to the error between the computed and desired output values. For the training process a set of feature vectors corresponding to each pattern class is used. Each training pattern consists of a pair of input and the corresponding target output. The patterns are given to the network consecutively, in an iterative manner. The appropriate weight corrections are made during training to adjust the network to the desired behavior. The iteration continues until the weight values allow the network to perform the required mapping. Each iteration of whole pattern set is named as an epoch.

Present work investigates the recognition capabilities of the FFMLP based Malayalam consonant recognition system using Multi Layer Feed Forward Neural Network (MLFFNN) and BP algorithm. The number of nodes in the input layer is fixed to 20 according to NWHF vector size. The number of nodes in the output layer is 36 for 36 Malayalam CV units. The experiment is repeated by changing the number of hidden layers. After trial and error experiments the number of hidden layer is fixed as 8 and the number of epochs as 10,000 for obtaining the successful architecture in the present study.

The simulation experiments and the results obtained using these two pattern recognition (ANN and k - NN ) approaches are presented in the next section.

\section{SIMULATION EXPERIMENT AND RESULTS}

In order to investigate the use of wavelet based $\mathrm{CV}$ speech unit feature enhancement and to compare with different speech modeling algorithms explained in section $\mathrm{V}$ with respect to their simulation experimental results in various noise levels, we carry out all of the above techniques in a noisy as well as clean speech recognition experiment and is summarized in the following para.

36 Malayalam basic consonant units from the speaker independent Malayalam CV speech unit corpus are used as the speech database for the present speech recognition task. Each $\mathrm{CV}$ speech signal lowpass filtered to $4 \mathrm{kHz}$ and sampled at $8 \mathrm{kHz}$ sampling rate and quantized to 16 bits is used in this study. The database consists of the utterances from 96 different native Malayalam speaking male and female young speakers. The dataset is then divided into training and testing set which contains first 48 samples for training and the remaining 48 samples for testing. Thus training and testing set contains a total of 1728 samples each. The main objective of this article is to design a robust $\mathrm{CV}$ speech unit recognizer in additive noisy condition. To perform this task each speech signal is corrupted by adding Additive White Gaussian Noise (AWGN) of different Signal to Noise (SNR) levels.

As explained in section III.1 an example of CWD based approximation signal plot is given in figure 3 ( $\mathrm{a}-$ e) . This represents $5^{\text {th }}$ level decomposition signal plot for the Malayalam CV speech databases of 5 different phonetic classes aspirated, unaspirated, nasals, approximants and fricatives.

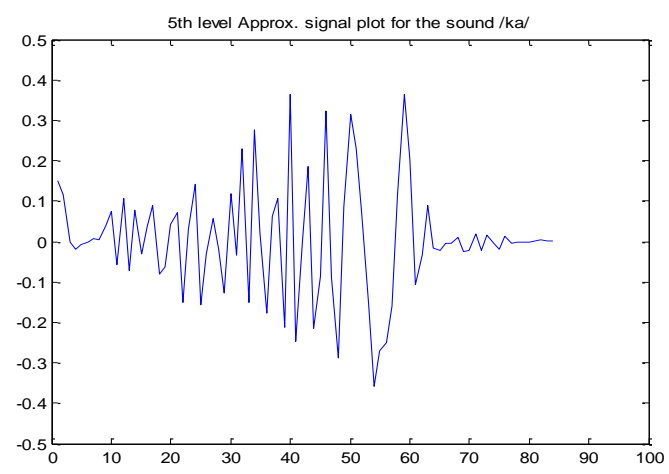

(a) 


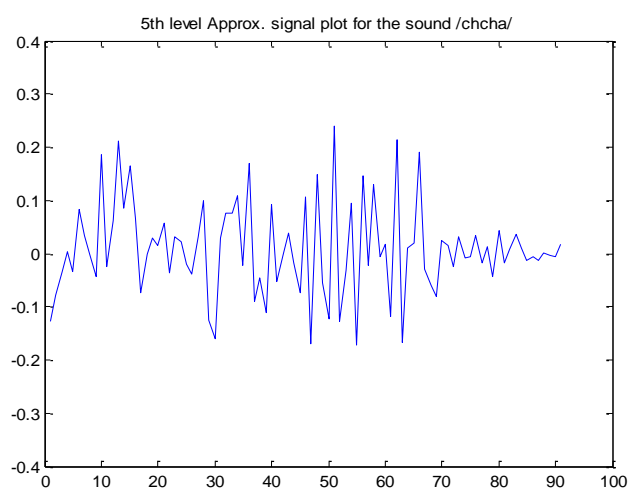

(b)

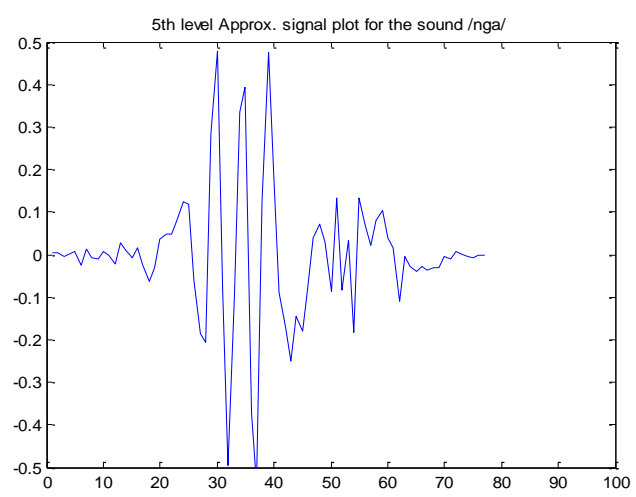

(c)

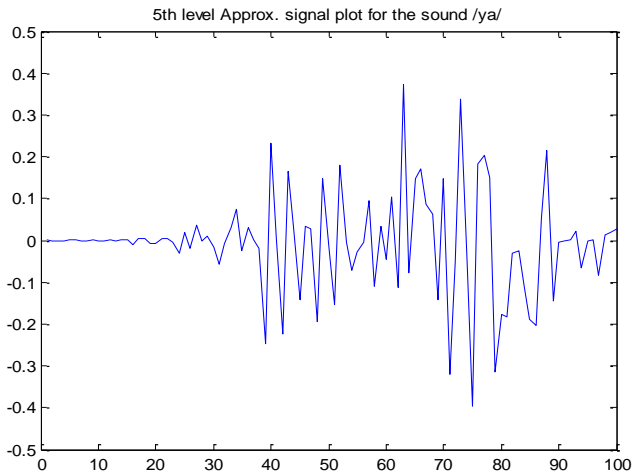

(d)

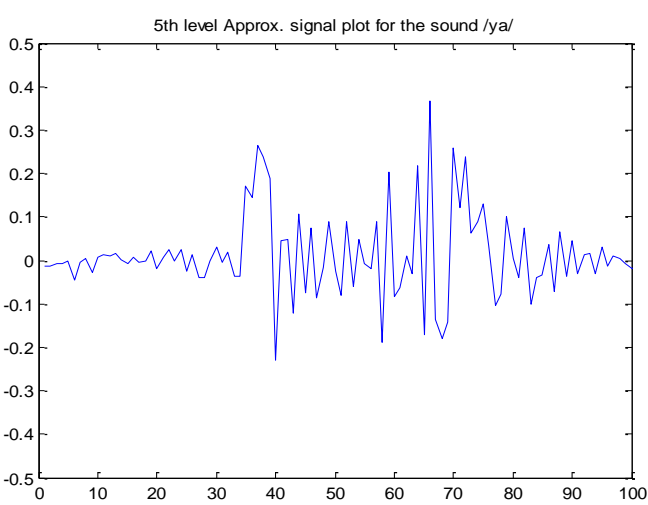

(e)

Figure 3. CWD based approximation signal plot for (a) Unaspirated, (b) Aspirated, (c) Nasals, (d) Approximants and (e) Fricatives
Section III.2 describes WPD coefficients for each CV speech sound for hybrid features. An example of WPD signal plot for 5 different phonetic classes are given in figure 4 .

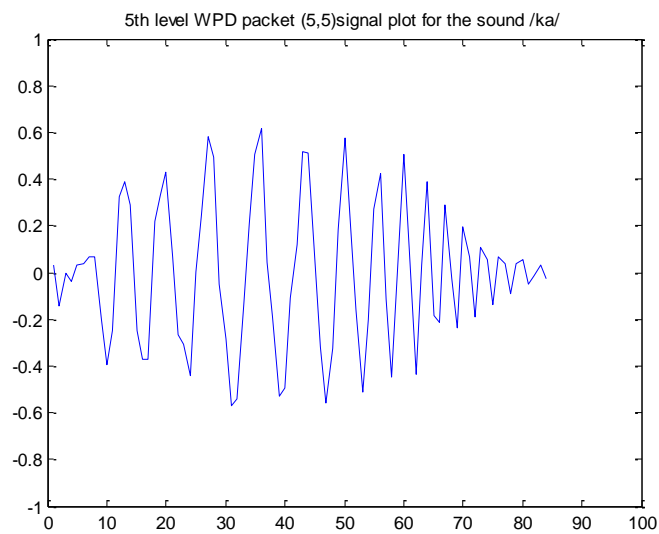

(a)

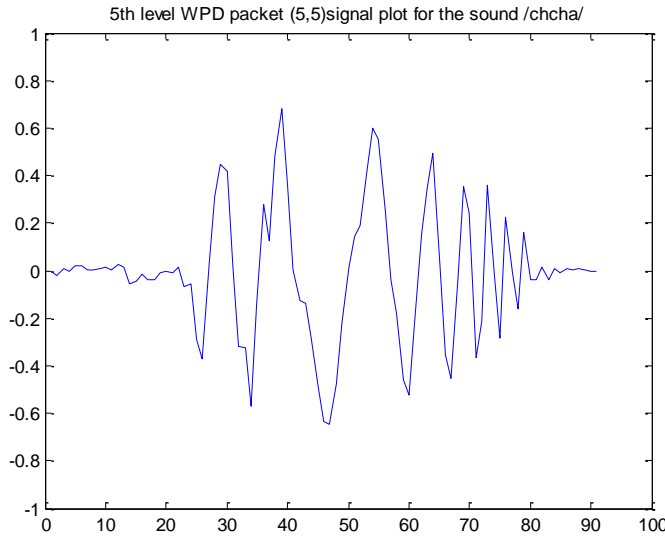

(b)

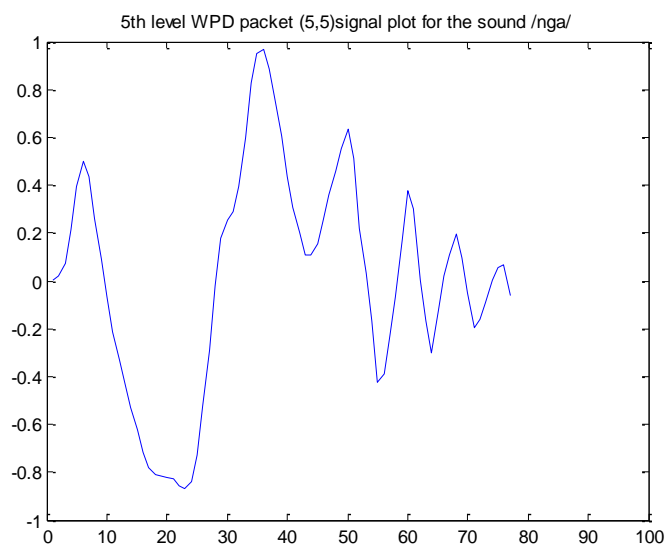

(c) 


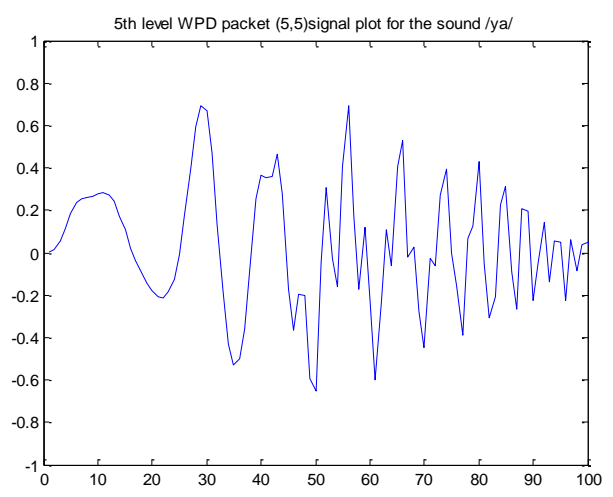

(d)

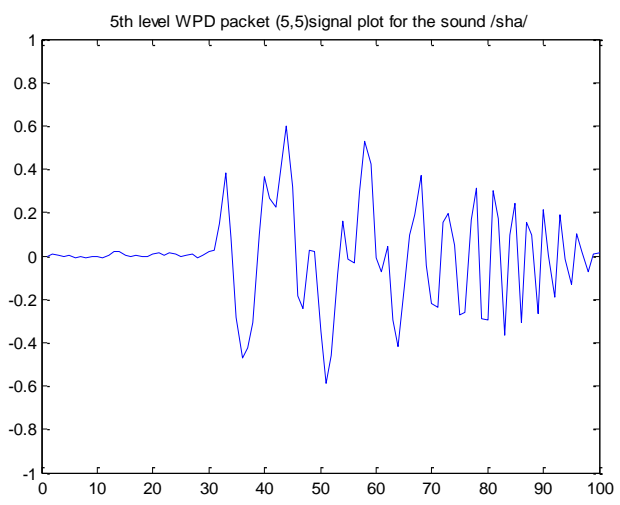

(e)

Figure 4. WPD signal plot for (a) Unaspirated, (b) Aspirated, (c) Nasals, (d) Approximants and (e) Fricatives
Considerable variation on these plots illustrates the difference in sound classes or groups under consideration for classification. Hence the NWHF feature vectors can effectively be used as a discriminate parameter for the classification purpose. Classification experiments are done using ANN and $\mathrm{k}$ - NN classifier. The simulation experiments are repeated using 5 different mother wavelets namely haar, symlets, coiflets, $\mathrm{db} 2$ and $\mathrm{db} 4$. It is found that Daubechies wavelet $\mathrm{db} 2$ is optimum for the classification of Malayalam CV speech unit database. Experimental results using 5 different wavelet families for clean speech using $\mathrm{k}-\mathrm{NN}$ and $\mathrm{ANN}$ are tabulated in Table 2 and Table 3 respectively.

The recognition accuracies obtained for Malayalam $\mathrm{CV}$ speech database for the five different phonetic classes at various SNR levels using $\mathrm{db} 2$ are tabulated in Table 4 and Table5.

Table 2: Recognition Accuracies for 5 different mother wavelets using NWHF vector and $\mathrm{k}-\mathrm{NN}$.

\begin{tabular}{|c|c|c|c|c|c|}
\hline Class & haar & symlets & Coiflets & $\mathrm{db} 2$ & $\mathrm{db} 4$ \\
\hline Unaspirated & 34.2 & 44.2 & 51.3 & 62.6 & 60.2 \\
\hline Aspirated & 36.1 & 45.9 & 50.5 & 63.3 & 60.9 \\
\hline Nasals & 37.8 & 43.8 & 49.3 & 65.7 & 62.4 \\
\hline Approximants & 35.3 & 46.9 & 52.9 & 64.9 & 61.5 \\
\hline Fricatives & 36.9 & 47.2 & 54.8 & 63.5 & 60.2 \\
\hline
\end{tabular}

Table 3: Recognition Accuracies for 5 different mother wavelet using NWHF vector and ANN.

\begin{tabular}{|c|c|c|c|c|c|}
\hline Class & haar & symlets & Coiflets & db2 & db4 \\
\hline Unaspirated & 45.9 & 54.8 & 62.3 & 71.8 & 70.1 \\
\hline Aspirated & 46.8 & 55.2 & 60.7 & 73.9 & 70.9 \\
\hline Nasals & 47.2 & 53.2 & 59.3 & 79.7 & 64.4 \\
\hline Approximants & 45.9 & 56.9 & 63.2 & 74.9 & 61.4 \\
\hline Fricatives & 46.1 & 57.9 & 64.6 & 76.4 & 63.7 \\
\hline
\end{tabular}

Table4. Recognition Accuracies of 5 different phonetic classes using NWHF and k - NN for different SNR values.

\begin{tabular}{|c|c|c|c|c|c|c|c|}
\hline Class & $0 \mathrm{~dB}$ & $3 \mathrm{~dB}$ & $10 \mathrm{~dB}$ & $20 \mathrm{~dB}$ & $30 \mathrm{~dB}$ & $40 \mathrm{~dB}$ & Clean \\
\hline Unaspirated & 25.7 & 28.9 & 45.5 & 56.2 & 62.4 & 62.6 & 62.6 \\
\hline Aspirated & 29.1 & 36.4 & 47.9 & 59.6 & 63.9 & 63.4 & 63.3 \\
\hline Nasals & 46.1 & 50.9 & 57.6 & 61.2 & 65.7 & 65.7 & 65.7 \\
\hline Approximants & 41.7 & 48.3 & 55.2 & 60.6 & 64.8 & 64.8 & 64.9 \\
\hline Fricatives & 43.3 & 49.1 & 56.5 & 60.9 & 63.1 & 63.6 & 63.5 \\
\hline
\end{tabular}


Table5 Recognition Accuracies of 5 different phonetic classes using NWHF and ANN for different SNR values.

\begin{tabular}{|c|c|c|c|c|c|c|c|}
\hline Class & $0 \mathrm{~dB}$ & $3 \mathrm{~dB}$ & $10 \mathrm{~dB}$ & $20 \mathrm{~dB}$ & $30 \mathrm{~dB}$ & $40 \mathrm{~dB}$ & $\mathrm{Clean}$ \\
\hline Unaspirated & 34.7 & 40.6 & 51.5 & 67.8 & 71.2 & 71.8 & 71.8 \\
\hline Aspirated & 36.9 & 42.7 & 54.8 & 70.2 & 73.8 & 73.9 & 73.9 \\
\hline Nasals & 44.1 & 51.3 & 58.7 & 74.8 & 79.9 & 79.6 & 79.7 \\
\hline Approximants & 40.4 & 46.9 & 55.4 & 71.6 & 74.5 & 74.9 & 74.9 \\
\hline Fricatives & 42.5 & 50.2 & 57.3 & 73.6 & 76.1 & 76.2 & 76.4 \\
\hline
\end{tabular}

\section{CONCLUSION}

A Multi Resolution Analysis (MRA) approach to Malayalam Consonant - Vowel (CV) speech unit recognition using Wavelet Transform (WT) has been studied. Two decomposition algorithms namely Classical Wavelet Decomposition (CWD) and Wavelet Packet Decomposition are combined to extract Normalized Wavelet Hybrid Feature (NWHF) vector for the present classification study. Daubecheis wavelet $\mathrm{db} 2$ is found as optimum mother wavelet in the present work. The recognition accuracies are calculated and compared using Artificial Neural Network (ANN) and k - Nearest Neighborhood $(\mathrm{k}-\mathrm{NN})$ at different levels of Signal to Noise Ratio (SNR) values and it is observed that the NWHF parameters improve their recognition accuracies by increasing SNR levels. Recognition of Malayalam $\mathrm{CV}$ speech unit with large database and effective implementation of wavelet features in combination with frequency domain features would be some of our future research directions.

\section{REFERENCES}

[1] Forgie J W and Forgie C D, "Results obtained from a Vowel Recognition Computer Program", Journal of Acoustical Society of America, Vol. 31, pp. 1480 - 1489, 1959.

[2] Reddy D R, "An approach to Computer speech recognition by Direct Analysis of the speech wave", Computer Science Dept., Stanford University Technical Report No. C549, 1966.

[3] Gold B and Morgan N, "Speech and Audio Signal Processing”, New York: John Wiley \& Sons Inc., 2000.

[4] Peter Ladefoged, " Vowels and Consonants- an Introduction to the Sounds of Language", BlackWell Publishing, 2004.

[5] Danial Jurafsky, James H Martin, "An Introd uction to Natural Language Processing, Computational Linguistics, and Speech Recognition", Pearson Education, 2004

[6] Hem P Ramachandran. (2008). Encyclopedia of Language and Linguistics. Pergamon Press: Oxford.

[7] H. Bourlard and S. Dupont, "Subband-based speech recognition," in Proceedings of IEEE International Conference on Acoustics, Speech, and Signal Processing (ICASSPSignal Processing (ICASSP '97), vol. 2, pp. 1251-1254, Munich, Germany, April 1997.
[8] M. Gupta and A. Gilbert, "Robust speech recognition using wavelet coefficient features," in Proceedings of IEEE Workshop on Automatic Speech Recognition and Understanding (ASRU '01), pp. 445-448, Madonna di Campiglio, Trento, Italy, December 2001.

[9] R. Sarikaya, B. L. Pellom, and J. H. L. Hansen, "Wavelet packet transform features with application to speaker Identification," in Proceedings of the 3rd IEEE Nordic Signal Processing Symposium(NORSIG '98), pp. 81-84, Vigs $\emptyset$, Denmark, June 1998.

[10] A Grossman, J Morlet \& P Gaoupillaud, "Cycle octave and related transforms in seismic signal Analysis", Geoexploration, Vol. 23, pp. 85-102, 1984.

[11] C torrence and G P Compo, "A practical guide to Wavelet Analysis", Bull. Amer. Meteorological. Soc., Vol. 79(1), pp. 61-78, 1998.

[12] Hongyu Liao, Mrinal Kr and Bruce F Cockburn, "Efficient Architectures for $1-\mathrm{D}$ and $2-\mathrm{D}$ Lifting Based Wavelet Transform", IEEE Trans. on Signal Processing, Vol. 52(5), pp. 1315 - 1326, 2004.

[13] S Mallat, "A wavelet Tour of Signal Processing, The Sparse Way", NewYork : Academic, 2009.

[14] K P Soman and K I Ramachandran, "Insight into Wavelets, from Theory to Practice", Prentice Hall of India, 2005.

[15] S Mallat, "A Theory for Multi resolution Signal Decomposition : The Wavelet Representation", IEEE Trans. on Pattern Analysis and machine Intelligence, Vol. 11, pp. 674 - 693, 1989.

[16] R Kronland - Martinet, J Morlet and A Grossman, "Analysis of Sound Patterns through Wavelet Transforms", Int. Journal of Pattern Recognition, Artificial Intelligence, Vol. 1(2), pp. 273 - 302 , 1987.

[17] R R Coifman and M Maggioni, "Diffusion Wavelets", Appl. Computat. Harmon. Anal, Vol. 21(1), pp. 53 - $94,2006$.

[18] D K Hammond, P Vandergheynst and R Gribonval, "Wavelets on Graph via Spectral Graph Theory, Appl. Computat. Harmon. Anal, 2010.

[19] Kadambe S and Boudreaux - Bartels G F, "Application of the Wavelet Transform for Pitch Detection of Speech Signal", IEEE Trans. on Information Theory, Vol. 38, pp. 917 - 924, 1992. 
[20] O Farook, S Dutta and M C Shrotriya, "Wavelet Sub band Based Temporal Features for Robust Hindi Phoneme Recognition", Int. Journal of Wavelets, Multi resolution and Information Processing (IJWMIP), Vol. 8(6), pp. 847 - 859, 2010.

[21] M Vetterly and C Herley, "Wavelets and Filter banks : Theory and Design", IEEE Trans. on Signal Processing, Vol. 40(9), pp. 2207 - 2232, 1992.

[22] M J Shensa, "Affine Wavelets: Wedding the Atrous and mallat Algorithms", IEEE Trans. on Signal Processing, Vol 40, pp. 2464 - 2482, 1992.

[23] S Mallat, " Multi frequency Channel Decomposition of Images and Wavelet Models", IEEE. Trans on Acoustics, Speech and Signal Processing, Vol. 37, pp. 2091 - 2110 , 1989.

[24] I Daubechies, "Ten Lectures on Wavelets", Philadelphia, PA:Soc. Appl. Math, 1992.

[25] Ronald W Lindsay, Donald B Percival and D Andrew Rothrock, "The Discrete Wavelet Transform and the scale analysis of the surface properties of Sea Ice", IEEE Trans. on Geo Science and Remote Sensing, Vol. 34(3), pp. 771 $787,1996$.

[26] P. J. Burt and E. H. Edelson, "The Lapalaciam Pyramid as a Compact Image Code", IEEE Trans. on Communications, Vol. COM-31, pp. $532-540$, 1983.

[27] J. Crowley, “A Representation for Visual Information”, Robotic. Inst. Carnegie-Mellon University, Tech. Rep. CMU - RI - TR - $82-7$, 1987.

[28] Yunlong Sheng, "Wavelet Transform-The Transforms and Application Handbook", CRC Press LLC, 2000.

[29] Duda . R. O and Hart P. E., " Pattern Classification and Scene Analysis", Wiley Inter Science, New York, 1973

[30] Duda R O, Hart P E and David G. Stork ,"Pattern Classification" A Wiley-Inter Science Publications, 2006.

[31] Tou J. T and Gonzalez R. C, "Pattern Recognition Principles", Addison - Wesley, London, 1974.

[32] Friedmen $M$ and Kandel $A$, "Introduction to Pattern Recognition: Statistical, Structural, Neural and Fuzzy Logic Approach", World Scientific, 1999.

[33] Cover T M \& Hart P E, "Nearest Neighbor Pattern Classification", IEEE trans. on Information Theory, Vol. 13 (1), pp. 21 - 27, 1967.

[34] Min-Chun Yu, " Multi - Criteria ABC analysis using artificial - intelligence based classification techniques", Elsevier - Expert Systems With Applications, Vol. 38, pp. 3416 - 3421, 2011.

[35] Hand D J, "Discrimination and classification", NewYork, Wiley, 1981

[36] Ray A. K and Chatterjee B, "Design of a Nearest Neighbor Classifier System for Bengali Character
Recognition", Journal of Inst. Elec. Telecom. Eng, Vol. 30, pp 226 - 229, 1984.

[37] Zhang. B and Srihari S N, "Fast k - Nearest Neighbor using Cluster Based Trees", IEEE trans. on Pattern Analysis and Machine Intelligence, Vol. 26(4), pp. 525 - 528, 2004.

[38] Pernkopf. F, "Bayesian Network Classifiers versus selective k -NN Classifier", Pattern Recognition, Vol. 38, pp. 1 - 10, 2005.

[39] Ripley. B. D, "Pattern Recognition and Neural Networks", Cambridge University Press, 1996.

[40] Haykin S, "Neural Networks: A Comprehensive Foundation", Prentice Hall of India Pvt. Ltd, 2004

[41] Simpson. P. K, "Artificial Neural Systems", Pergamon Press, 1990.

[42] W S McCullough \& W H Pitts, " A logical calculus of ideas immanent in nervous activity", Bull Math Biophysics, Vol 5, pp. 115-133, 1943

[43] R P Lippmann, "An introduction to computing with Neural Nets", IEEE Trans. Acoustic Speech \& Signal Processing Magazine., Vol 61., pp 4 $22 ., 1987$.

[44] T Kohonen, "An introduction to Neural Computing, Neural Networks, 1988.

[45] Sankar K Pal \& Sushmita Mitra, "Multilayer perceptron, Fuzzy sets, and Classification”, IEEE Trans. Neural Networks., Vol 3(5)., 1992.

T M Thasleema, female, is a Research Scholar at the Department of Information Technology, Kannur University. She had to her credit two book chapters and many research publications in national and international levels in the area of speech processing and pattern recognition. Currently she is doing her Ph.D in speech signal processing at Department of Information Technology, Kannur University under the supervision of Prof Dr N. K Narayanan.

N K Narayanan, male, is a Senior Professor of Information Technology, Kannur University, Karala, India. He earned a Ph.D in speech signal processing from Department of Electronics, CUSAT, Kerala, India in 1990. He has published about eighty four research papers in national $\&$ international journals in the area of Speech processing, Image processing, Neural networks, ANC and Bioinformatics. He has served as Chairman of the School of Information Science \& Technology, Kannur University during 2003 to 2008, and as Principal, Coop Engineering College, Vadakara, Kerala, India during 2009-10. Currently he is the Director, UGC IQAC, Kannur University. 